\title{
Bilateral pathological neck of femur fractures associated with low grade splenic marginal zone lymphoma: Case report, and review of the literature
}

\author{
F Fallahi ${ }^{1}$, A Waton ${ }^{1}$, M Mansha ${ }^{1}$, W Cope $^{2}$, H O'Brien ${ }^{1}$, C F Young $^{1}$ \\ 1. Cumberland Infirmary, Carlisle, Cumbria CA2 7HY, UK. 2. Royal Victoria Infirmary, Newcastle Upon Tyne, UK.
}

Correspondence: Farshid Fallahi. Address: Cumberland Infirmary, Newtown Road, Carlisle, CA27HY, UK. Email: farshid.fallahi@ncuh.nhs.uk

Received: February 7, 2014

Accepted: April 4, 2014

Online Published: April 20, 2014

DOI : $10.5430 /$ ijdi.v1n2p47

URL: http://dx.doi.org/10.5430/ijdi.v1n2p47

\section{Abstract}

We report a case involving a 64 year old lady with bilateral pathological fractures of the neck of the femur secondary to Low Grade Splenic Marginal Zone Lymphoma, a pathology that has not previously been described in clinical literature. This particular case also highlights the value of the diagnosis and management of patients presenting with subtle and unusual symptoms, and supports the current guidance of the National Institute for Health and Care Excellence regarding the investigation of patients with suspected pathological neck of femur fracture.

\section{Keywords}

Bilateral pathological hip fracture, MRI hip, Splenic marginal zone lymphoma, B-cell lymphoma

\section{Introduction}

Splenic Marginal Zone Lymphoma (SMZL) is a B-cell lymphoma that is recognised within the classification of Tumours of Haematopoietic and Lymphoid tissues of the World Health Organisation. It is characterised by splenomegaly and bone marrow involvement. Further characteristic features are moderate lymphocytosis and relative indolent course with no evidence of active disease in $80 \%$ of cases ${ }^{[1]}$. Tumour progression and aggressive behaviour are observed in a minority of patients ${ }^{[1]}$. Spread to extra-nodal sites is rare and often associated with aggressive disease; spread to pleura, skin, and meninges has been described ${ }^{[2]}$.

Bilateral fractures of the neck of the femur are uncommon, but well reported in the literature. They can occur following major or minor trauma ${ }^{[3,4]}$, seizures and convulsive therapy ${ }^{[5,6]}$. They can also occur due to primary or secondary bone pathology ${ }^{[7]}$, most commonly metastatic spread, or multiple myeloma ${ }^{[8,9]}$. Sequential bilateral fractures are more common than concurrent fractures, and are often secondary to simple mechanical falls in elderly patients with osteoporosis ${ }^{[3,7]}$.

To our knowledge there are no clinical reports of pathological fracture associated with SMZL available in the public domain.

We report a case of a patient who sustained bilateral fractures of the neck of the femur associated with SMZL, and highlight the pertinence of current guidance of the National Institute for Health and Care Excellence (NICE) regarding follow up imaging in cases of suspected pathological fracture where initial plain-film radiographs do not show a fracture. 


\section{Case report}

A 53 year old lady underwent a Splenectomy for splenomegaly in February 1998. The size of spleen was measured at 19 $\mathrm{cm} \times 3 \mathrm{~cm} \times 8 \mathrm{~cm}$. The histology demonstrated congestion but showed no evidence of any further abnormalities. The patient had an uneventful post-operative course and had no further follow up.

She presented 11 years later at the age of 64 with aplastic anaemia. She reported one week of left thigh pain with no history of trauma. She complained of a dull ache and was found to have pain on internal rotation of the hip. Plain film radiographs were unremarkable (see Figure 1). CT (Siemens Somaton Sensation 64, Siemens, Camberley, UK) of chest, abdomen and pelvis was done to look for intra-abdominal pathology, specifically lymphadenopathy but found no abnormality other than splenectomy. Proximal femora were imaged within this study and showed no evidence of fracture (see Figure 2). MRI (Siemens 1.5 Tesla Avanto, Siemens, Camberley, UK) of the spine did not include hips, but reported "Diffusely abnormal marrow signal throughout the spine, presumably related to aplastic anaemia.” Radioisotope bone scan was also done, and showed no increased uptake within the proximal femur (see Figure 3).

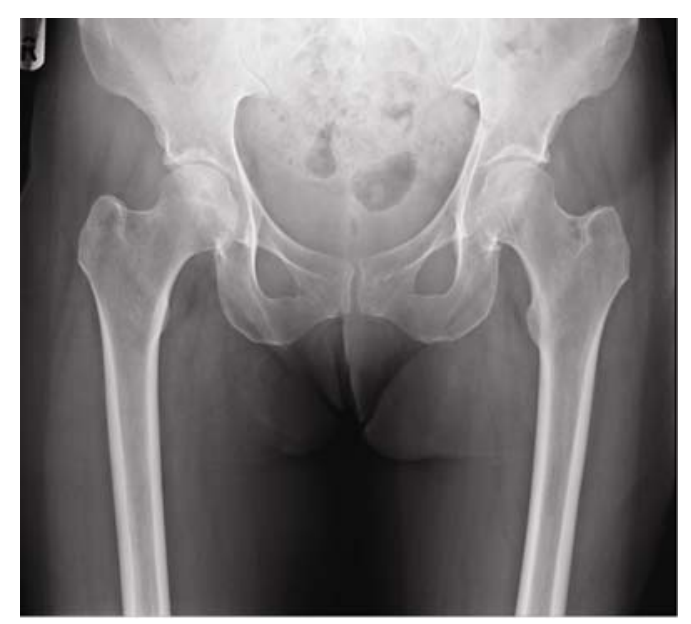

Figure 1. AP pelvis radiograph during initial admission. No fracture is seen.

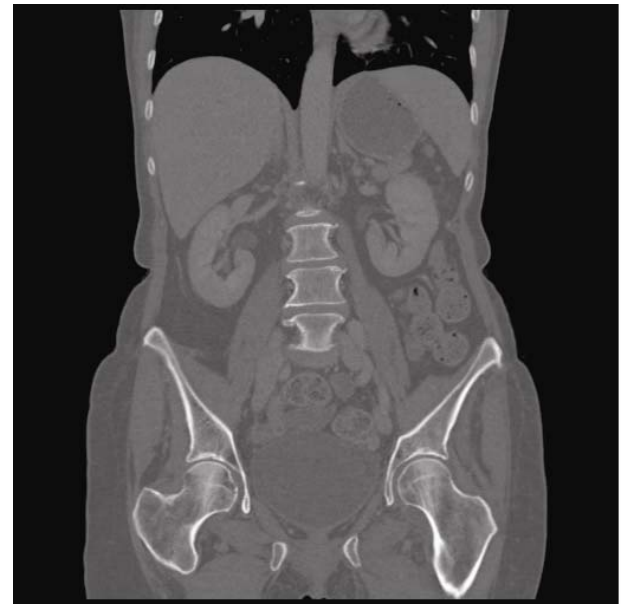

Figure 2. Coronal CT image including femoral neck fails to demonstrate a fracture

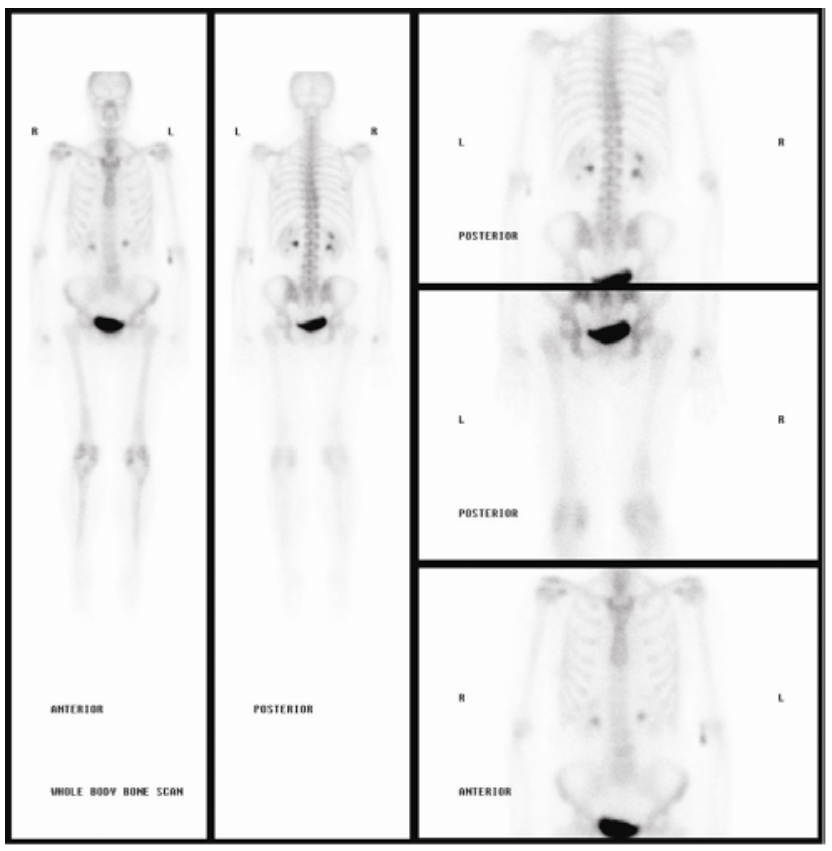

Figure 3. Radioisotope bone scan performed during initial presentation. No increased uptake is demonstrated in proximal femur. 
She was discharged home. The patient was re-admitted 6 weeks later with neutropaenic sepsis and right knee pain with a fixed flexion deformity. She was found to have septic arthritis of the right knee.

While she was mobilising on the ward a few days later she heard a "crack" from her left hip and was unable to weight bear. Radiographs showed a displaced subcapital fracture of the neck of the femur on the left (see Figure 4).

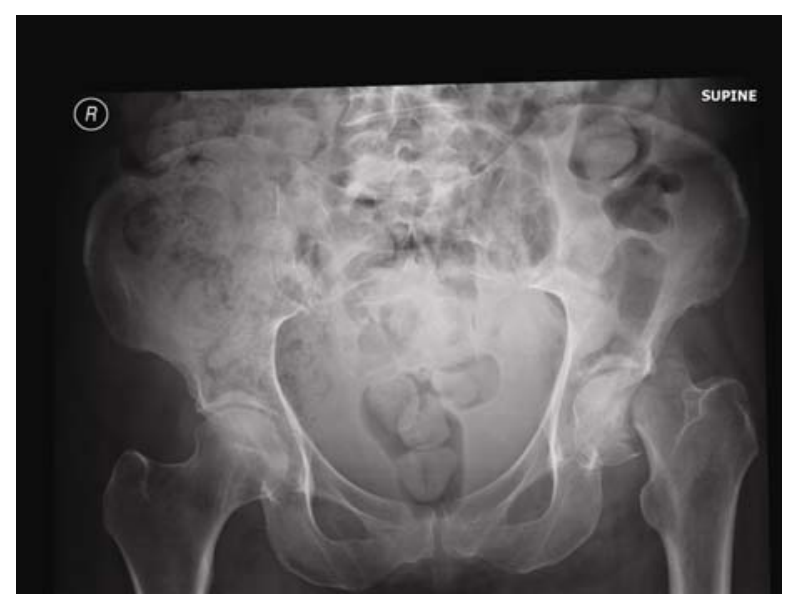

Figure 4. AP pelvis radiograph at subsequent presentation demonstrating a displaced subcapital fracture of the neck of femur on the left. No fracture seen on the right.

MRI scan (Siemens 1.5 Tesla Avanto, Siemens, Camberley, UK) was obtained due to the uncertain history of haematological malignancy, and the atraumatic mechanism of injury. The MRI showed a displaced pathological fracture of the neck of the left femur with patchy bone marrow changes, as well as an undisplaced pathological fracture of the head and neck of the right femur with patchy bone marrow changes (see Figures 5 and 6). Of note, the right hip was not painful at any stage.

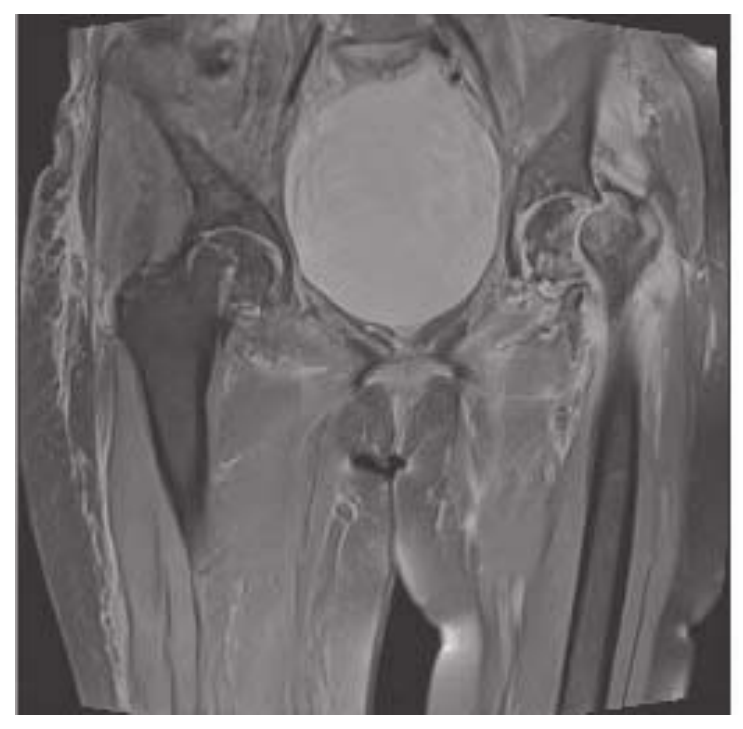

Figure 5. Coronal PDFS image showing bone marrow changes around the fracture of neck of femur on the left. Note made of an undisplaced fracture of the right femur.

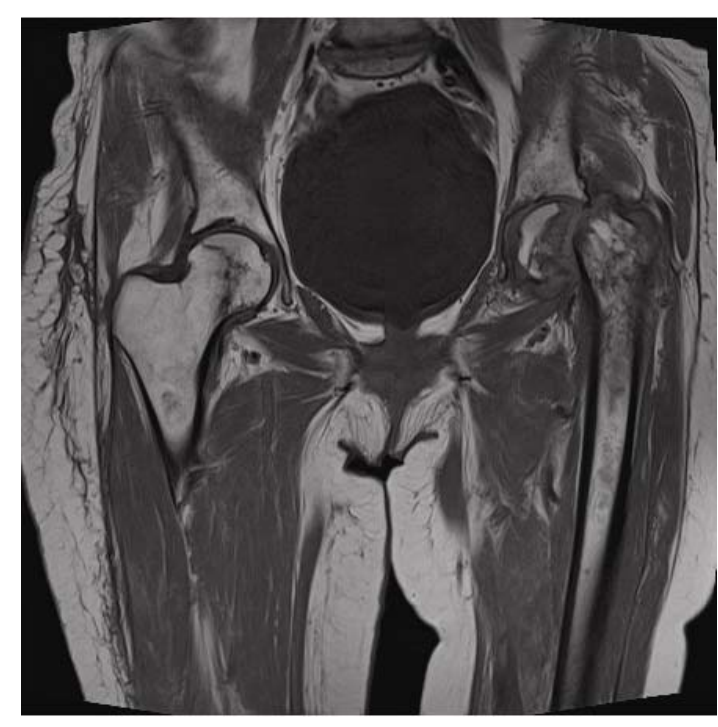

Figure 6. Coronal $\mathrm{T} 1$ weighted image showing bone marrow changes around the fracture of the left femur, and the undisplaced fracture of the right femur. 
Bone marrow biopsy was performed in theatre, and histology showed lymphoid aggregates consistent with splenic marginal zone lymphoma. No evidence of high grade lymphoma or metastatic malignancy was seen (see Figure 7).

Further review of the histology from her splenectomy at this point (2009, RVI, Newcastle, UK and Centro Nacional de Investigationes Oncologicas, Madrid, Spain) confirmed low-grade Splenic Marginal Zone Non-Hodgkins Lymphoma with an interstitial and nodular pattern (see Figures 8, 9 and 10).

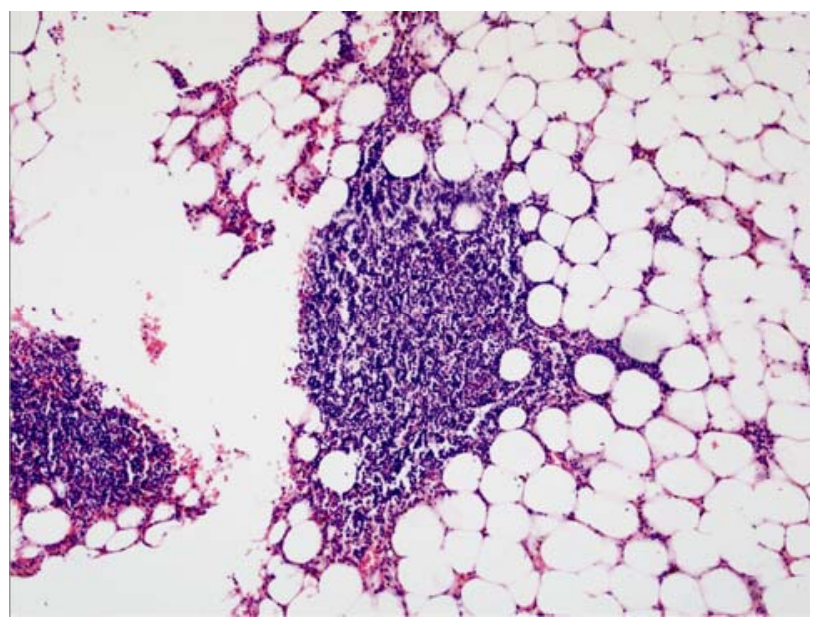

Figure 7. Bone fragments $(\mathrm{H} \& \mathrm{E}, \times 10)$ : Hypocellular marrow, with most of the more cellular areas being areas of fat necrosis or prominent lymphoid aggregates, consistent with the known splenic marginal zone lymphoma

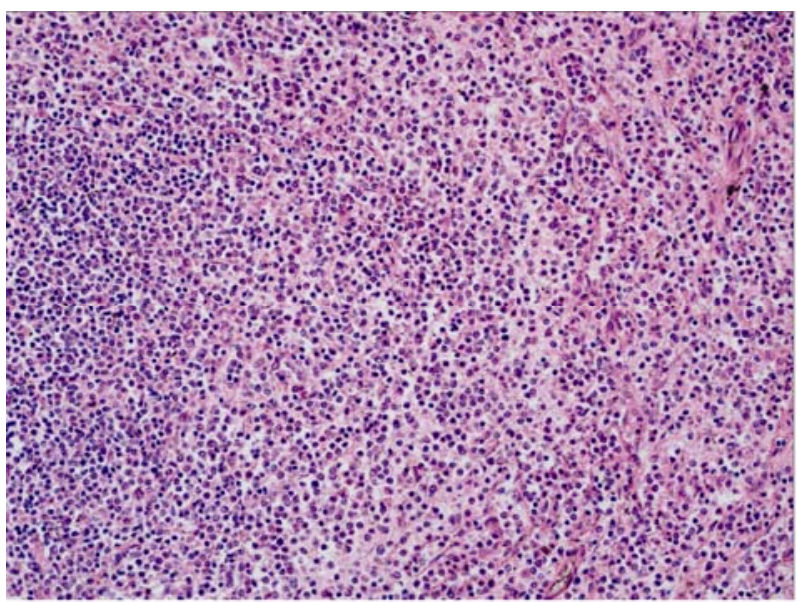

Figure 9. Spleen $(H \& E, \times 20)$ : The infiltrate comprises predominantly of small lymphoid cells.

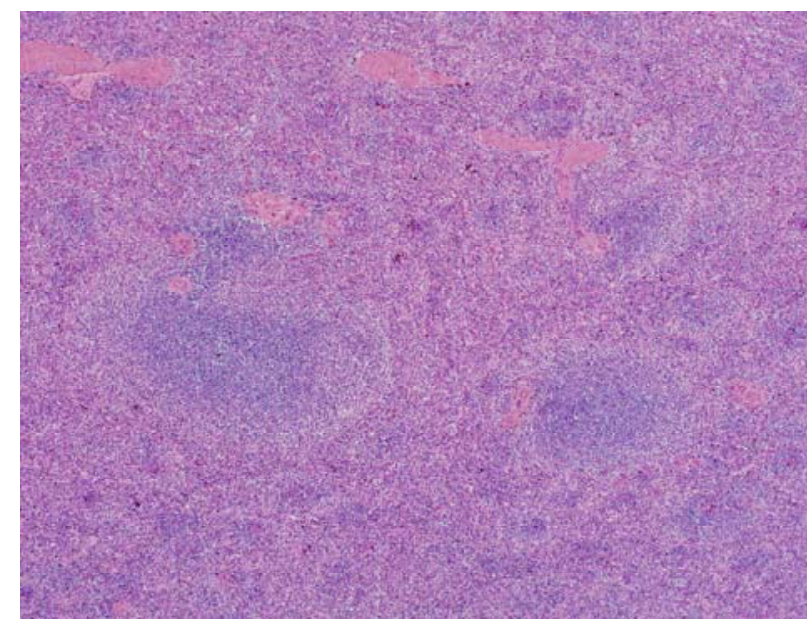

Figure 8. Spleen $(\mathrm{H} \& \mathrm{E}, \quad \times 4)$ : abnormal lymphoid proliferation within both red and white pulp components.

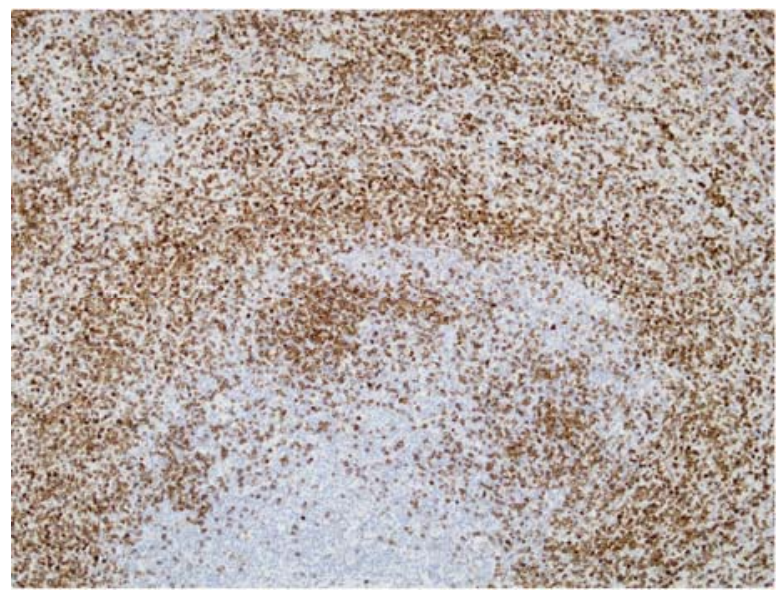

Figure 10. Spleen (CD79a): The infiltrate comprises mainly of B cells. The following were performed but not shown here: positive for CD20, Bcl2 and IgM; negative for CD5, Cd43, cyclin D1, Bcl6, CD10, CD23, CD25 and annexin A1; Ki67 proliferation fraction is low.

She underwent a long-stemmed bipolar hemiarthroplasty of the left hip a few days later and made an uneventful recovery from this. She had no operative intervention for her right hip.

Unfortunately, her aplastic anaemia did not improve, and the patient went into bone marrow failure. Despite treatment she died from neutropaenic sepsis 13 months after the hip hemiarthroplasty. 


\section{Discussion}

Bilateral fractures of the neck of the femur are a rare, but well described clinical entity. They are associated with a significant increase in mortality compared to unilateral neck of femur fractures ${ }^{[3]}$. Bilateral pathological fractures have been reported due to hypocalcaemia, osteomalacia, osteoporosis, renal osteodystrophy, metastatic disease, lymphoma, multiple myeloma, as well as persistent stress ${ }^{[4,7,9]}$.

To our knowledge no case of pathological fracture secondary to SMZL has yet been reported in the literature.

Secondary bone lymphoma by definition is involvement of bone in the presence of primary lesion somewhere else. In a study of 422 patients with malignant lymphoma of the bone, Ostrowski et al ${ }^{[10]}$ defined primary bone lymphoma (PBL) as a tumour presenting in an osseous site with no evidence of disease elsewhere for at least 6 months after diagnosis. The PBL can spread to regional nodes and distant sites particularly to reticuloendothelial system ${ }^{[8]}$. It seems clear in this case however, that this was a secondary bone lymphoma, which spread slowly from the primary SMZL, presenting some 11 years later.

Pathological fracture is a recognized complication of both primary and secondary bone lymphoma but is uncommon. In a large series of 131 patients with primary bone lymphoma over a period of 22 years, one third had lymphoma involvement of the long bones with pathological fracture occurring in only nine patients ${ }^{[5]}$. In another retrospective analysis of 36 patients with primary $(n=17)$ and secondary $(n=19)$ bone lymphoma surgically treated at an orthopaedic centre over a period of 15 years, pathological fracture of the proximal femur or humerus was observed in three patients ${ }^{[6]}$.

Our patient sustained pathological fracture secondary to low grade SMZL involvement of the femur. This is uncommon even in patients with lymphoma involvement of the long bones ${ }^{[11]}$. Furthermore, she presented with pain in her hip and thigh which is an unusual symptom of bone involvement in a patient presenting with non-Hodgkin's lymphoma (NHL) ${ }^{[12]}$. However, this is a common presentation of pathological hip fracture. There were no enlarged lymph nodes on clinical examination or on CT staging scan. While splenic hilar lymphadenopathy is common in SMZL, peripheral lymph node involvement is less common ${ }^{[1]}$. Chacon et al's reported peripheral lymphadenopathy was only present in $17 \%$ of 60 patients with SMZL ${ }^{[2]}$. This clinical manifestation was probably one of the reasons behind the delay in diagnosis in this case.

The earliest radiological sign of bone involvement in lymphoma is diffuse medullary destruction - a 'moth-eaten' appearance with poorly defined margins. At this stage it is impossible to differentiate the lesion from other neoplastic or infective processes. A periosteal reaction is rare. Cortical destruction occurs eventually and a surrounding soft tissue mass ensues. Sclerotic lesions are uncommon ${ }^{[13]}$. The delay in diagnosis of this fracture was also attributable to the radiological appearances. The initial X-ray, CT scan and radioisotope bone scan did not show any suspicious lesions. The second X-ray 6 weeks later showed some lytic lesions in the intertrochanteric region just below the fracture, but no other abnormalities. However, localised MRI showed involvement of the bone marrow in both femora (see Figures 1 to 6 above).

There is limited evidence in the literature comparing CT, radioisotope, and MRI scanning in the diagnosis of hip fracture not visualised on plain films. Two studies report a sensitivity of $75-98 \%$ and specificity of $100 \%$ for bone scan compared to MRI ${ }^{[13,14]}$. These studies were limited by their methodologies, added to this, radioisotope bone scans take longer to acquire than MRI. Two small studies (17 patients in total) comparing CT and MRI for the diagnosis of occult hip fracture have both recommended that MRI is superior ${ }^{[15,16]}$.

It is also notable that while CT and radioisotope scans both expose the patient to ionising radiation, MRI does not.

A review article considering the choice of imaging in occult hip fracture recommends MRI if plain films are not diagnostic and comments on the lack of evidence supporting the use of $\mathrm{CT}^{[17]}$. 
NICE guidance published in June 2011 also recommends MRI as the gold standard investigation for occult hip fracture, citing greater diagnostic accuracy than radioisotope scanning or CT, as well as not exposing the patient to ionising radiation. However, CT is an acceptable alternative in situations where there is limited availability of MRI, or where its use is contraindicated ${ }^{[18]}$.

This case supports the NICE guidance, as well as the recommendation from the current literature that MRI is the imaging modality of choice in patients with occult hip fractures not visualised on plain films. MRI should be considered in patients with persisting hip pain and clinical suspicion of fracture even when CT scan is negative.

\section{Conclusion}

This case report reiterates the importance of considering pathological fractures in high risk patients who present with musculoskeletal pain. Plain radiographs may be falsely reassuring, and a high index of suspicion is needed in patients with signs and symptoms suggestive of skeletal involvement.

It also supports the current NICE guidance by highlighting the relative shortcomings of CT and radioisotope bone scans in the diagnosis of pathological fracture, as both were normal at her initial presentation. Further imaging with MR scan is necessary to avoid delayed diagnosis and any ensuing morbidity.

\section{Acknowledgements}

We are indebted to Dr C Bacon, Consultant Histopathologist at The Royal Victoria Infirmary, Newcastle Upon Tyne, UK for his work reviewing all the histology for this case, as well as organising the review from the Centro Nacional de Investigationes Oncologicas, Madrid, Spain.

We would also like to thank the staff at the Centro Nacional de Investigationes Oncologicas, Madrid, Spain for their expert help.

\section{References}

[1] Franco V, Florena AM, Iannitto E. Splenic marginal zone lymphoma. Blood. 2003; 101-7: 2464-72.

[2] Chacón JI, Mollejo M, Muñoz E, Algara P, Mateo M, Lopez L, et al. Splenic marginal zone lymphoma: clinical characteristics and prognostic factors in a series of 60 patients. Blood. 2002; 100-5: 1648-5.

[3] Boston DA. Bilateral fractures of the femoral neck. Injury. 1982; 14-3: 207-10.

[4] Sood A, Rao C, Holloway I. Bilateral femoral neck fractures in an adult male following minimal trauma after a simple mechanical fall: a case report. Cases J. 2009; 2-1: 92.

[5] Kause J, Parr MJ. Bilateral subcapital neck of femur fractures after eclamptic seizures. Br J Anaesth. 2004; 92-4: 590-2. http://dx.doi.org/10.1093/bja/aeh105

[6] Powell HD. Stimultaneous bilateral fractures of the neck of the femur. J Bone Joint Surg Br. 1960; 42-B: $236-52$.

[7] Gaulke R. [Spontaneous bilateral fractures of the femoral neck. Etiology-frequency-diagnosis-therapy]. Z Orthop Ihre Grenzgeb 2002; 140-3: 297-303. http://dx.doi.org/10.1055/s-2002-32478

[8] Mouzopoulos G, Stamatakos M, Kouvaris C, Mouzopoulos D, Tzurbakis M. Spontaneous bilateral subcapital hip fracture due to multiple myeloma: an 18-month follow-up. Eur J Cancer Care (Engl). 2007; 16-5: 413-6.

[9] Jacofsky DJ, Haidukewych GJ. Management of pathologic fractures of the proximal femur: state of the art. J Orthop Trauma. 2004; 18-7: 459-69.

[10] Ostrowski ML, Unni KK, Banks PM, Shives TC, Evans RG, O'Connell MJ, et al. Malignant lymphoma of bone. Cancer. 1986; 58-12: 2646-55.

[11] Chim CS, Yiu CP, Shek WH. Pathological bone fracture in non-Hodgkin's lymphoma. J Clin Oncol. 2007 Jul 20; $25(21)$ :.3175-6. http://dx.doi.org/10.1200/JCO.2007.11.9966

[12] Falcini F, Bardare M, Cimaz R, Lippi A, Corona F. Arthritis as a presenting feature of non-Hodgkin's lymphoma. Arch Dis Child. 1998 Apr; 78(4): 367-70. http://dx.doi.org/10.1136/adc.78.4.367 
[13] Evans PD, Wilson C, Lyons K. Comparison of MRI with bone scanning for suspected hip fracture in elderly patients. J Bone Joint Surg Br. 1994; 76-1: 158-9.

[14] Rizzo PF, Gould ES, Lyden JP, Asnis SE. Diagnosis of occult fractures about the hip. Magnetic resonance imaging compared with bone-scanning. J Bone Joint Surg Am. 1993; 75-3: 395-401.

[15] Lubovsky O, Liebergall M, Mattan Y, Weil Y, Mosheiff R. Early diagnosis of occult hip fractures MRI versus CT scan. Injury. 2005; 36-6: 788-92.

[16] Deutsch AL, Mink JH, Waxman AD. Occult fractures of the proximal femur: MR imaging. Radiology. 1989; 170-1 Pt 1:113-6.

[17] Cannon J, Silvestri S, Munro M. Imaging choices in occult hip fracture. J Emerg Med. 2009; 37-2: 144-52.

[18] National Clinical Guideline Centre. [Internet] The Management of Hip Fracture in Adults. London: National Clinical Guideline Centre, 2011. www.ncgc.ac.uk. [updated 2011 Aug 11; Cited 2012 June 12] Available from:

http://www.nice.org.uk/nicemedia/live/13489/54918/54918.pdf 